\title{
Get Your Hands Off My Laptop: Physical Side-Channel Key-Extraction Attacks on PCs
}

\author{
Daniel Genkin ${ }^{1,2}$, Itamar Pipman ${ }^{2}$, and Eran Tromer ${ }^{2}$ \\ 1 Technion, Israel \\ danielg3@cs.technion.ac.il \\ 2 Tel Aviv University, Israel \\ \{itamarpi,tromer\}@tau.ac.il
}

\begin{abstract}
We demonstrate physical side-channel attacks on a popular software implementation of RSA and ElGamal, running on laptop computers. Our attacks use novel side channels, based on the observation that the "ground" electric potential, in many computers, fluctuates in a computation-dependent way. An attacker can measure this signal by touching exposed metal on the computer's chassis with a plain wire, or even with a bare hand. The signal can also be measured at the remote end of Ethernet, VGA or USB cables.

Through suitable cryptanalysis and signal processing, we have extracted 4096-bit RSA keys and 3072-bit ElGamal keys from laptops, via each of these channels, as well as via power analysis and electromagnetic probing. Despite the GHz-scale clock rate of the laptops and numerous noise sources, the full attacks require a few seconds of measurements using Medium Frequency signals (around $2 \mathrm{MHz}$ ), or one hour using Low Frequency signals (up to $40 \mathrm{kHz}$ ).
\end{abstract}

\section{Introduction}

\section{$1.1 \quad$ Background}

Side-channel attacks that exploit unintentional, abstraction-defying information leakage from physical computing devices have proven effective in breaking the security of numerous cryptographic implementations. However, most research attention has been focused on small devices: smartcards, RFID tags, FPGAs, microcontrollers, and simple embedded devices. The "PC" class of devices (commodity laptop/desktop/server computers) has been studied from the perspective of side channels measured by resident software (see [13] and subsequent works) and from peripherals (e.g., [17]).

PCs, however, have received little academic attention with regard to physical emanations from cryptographic computations, presumably due to three main barriers. First, PCs have highly complicated system architecture and CPU micro architecture, with many noise sources and asynchronous events. Fine lowlevel events are thus difficult to model and measure. Second, most physical 
side-channel cryptanalysis approaches require the leakage signal to be acquired at rates well beyond the device's clock rate; for multi-GHz CPUs, the requisite equipment is expensive, and the signals are difficult to probe. Finally, attack scenarios differ: the aforementioned small devices are often deployed into potentially-malicious hands, where they could be subjected to lengthy or invasive attacks; but for PCs, the typical scenario (short of theft) is where a physical attacker gains physical proximity for a restricted amount of time, and must operate surreptitiously.

Recently, a key extraction attack on PCs was demonstrated using the acoustic side channel, addressing all three barriers: using a chosen-ciphertext attack, the sound emanations of interest are made very robust, brought down to very low frequencies (tens or hundreds of $\mathrm{kHz}$ ), and extended to long durations (hundreds of milliseconds), making it possible to record the leakage surreptitiously and noninvasively, by a cellphone microphone or from many meters away [12].

We thus study the question: what other physical, non-invasive, cryptanalytic side-channel attacks can be effectively conducted on full-blown PC computers?

\subsection{Our Results}

We demonstrate key extraction of 4096-bit RSA and 3072-bit ElGamal keys from laptop computers of various models. The attacked software implementation is GnuPG [2], a popular open source implementation of the OpenPGP standard. The attacks exploit several side channels, enumerated below:

1. Chassis potential. We identify a new side channel: fluctuations of the electric potential on the chassis of laptop computers, in reference to the mains earth ground. This potential can be measured by a simple wire, non-invasively touching a conductive part of the laptop, and connected to a suitable amplifier and digitizer. (This, essentially, creates a ground loop through the laptop and measures its voltage.) The chassis potential, thus measured, is affected by ongoing computation, and our attacks exploit this for extracting RSA and ElGamal keys, within a few seconds.

Scenarios: The wire can be fixed in advance where the target laptop will be placed (e.g., a cluttered desk), or put in contact with the laptop by a passerby.

2. Far end of cable. The chassis potential can also be observed from afar, through any cable with a conductive shield that is attached to an I/O port on the laptop. For example, we demonstrated key recovery through a 10-meter long Ethernet cable, by tapping the cable shield at the remote Ethernet switch (see Figure 4(a). Similar observations apply to USB, VGA, HDMI, etc. Since only the shield potential is used, the attack is oblivious to the data passing through the cable, and works even if the port is disabled.

Scenarios: While many users are careful about connecting suspicious devices (such as USB sticks) to the physical ports of their machines, they will routinely connect VGA display cables and Ethernet network cables to their laptops. However, a simple voltage measurement device, perhaps located in the cabinet or server room to which the cable leads, could be capturing the 
leakage. This is hard to check, since Ethernet wiring and projectors' VGA cables are often hidden out of sight and cannot easily be tracked by the user.

3. Human touch. Surprisingly, the requisite signal can be measured, with sufficient fidelity, even through a human body. An attacker merely needs to touch the target with his bare hand, while his body potential is measured.

Scenarios: The attacker positions himself in physical proximity to the target laptop and touches it with his bare hand or a conducting pen (see Figures 4(b) 4(c). Surreptitiously, the attacker carries the requisite equipment for measuring his body potential relative to some nearby grounded object. In the non-adaptive attack (see below), a few seconds' contact will suffice; in the adaptive attack, 1 key bit can be extracted approximately every 4 seconds.

Note that the above attacks all rely on fluctuations in the PC's ground (relative to the mains earth ground). This makes mitigation difficult: the usual method for preventing voltage fluctuations, using bypass capacitors to shunt stray AC currents into the device's ground, does not apply to the device ground itself.

We also revisit two traditional physical side channels, and demonstrate their applicability to software running on PCs:

4. Electromagnetic (EM). We performed key extraction by measuring the induced EM emanations, using a near-field probe placed next to the laptop.

Scenarios: Electromagnetic probes are easily hidden in nearby objects. A glove, containing a concealed probe loop and hovering over the target laptop, would unveil its key within seconds.

5. Power. Likewise, we extracted keys by measuring the current draw on the laptop's power supply. Our attack works even though PCs use complex switching power supplies, which partially decouple the power source from the CPU load 1, and moreover employ large capacitors, chokes, and shields for electromagnetic compatibility (EMC) compliance - all of which attenuate and disrupt the signals sought in traditional power analysis.

Scenarios: A public charging station can be easily augmented with a current meter, logger, and transmitter. Even a regular power supply "brick" can be similarly augmented.

Our attacks require very low bandwidth, well below the laptop CPU's GHz-scale clock rate. We use two cryptanalytic approaches, based on known techniques and adapted to the target software:

Fast, Non-adaptive MF Attack. For both RSA and ElGamal key extraction, we can exploit signals circa $2 \mathrm{MHz}$ (Medium Frequency band), using the " $n-1$ " non-adaptive chosen-ciphertext simple-power-analysis attack of Yen et al. 26]. Key extraction then requires a few seconds of measurements.

Slow, Adaptive VLF/LF Attack. For RSA key extraction, we can exploit signals of about $15-40 \mathrm{kHz}$ (Very Low / Low Frequency bands), using

${ }^{1}$ In the realm of small devices, such similar decoupling has been proposed as an intentional countermeasure against power analysis [23]. 
the adaptive chosen-ciphertext attack of [12. Full 4096-bit RSA key extraction then takes approximately one hour, but is very robust to low signal-to-noise ratio.

Our results require careful choice and tuning of the signal acquisition equipment, to attain usable signal-to-noise ratio in the presence of abundant noise, delicate grounding, and impedance-matching considerations. We report these choices in detail and describe the requisite signal processing. We also analyze the code of GnuPG's mathematical library, showing why the specific chosen ciphertext creates exploitable, key-dependent leakage in this implementation.

\subsection{Vulnerable Software and Hardware}

Hardware. We have tested various laptop computers, of different models, by various vendors. The signal quality varied dramatically, as did the relative quality between channels, the carrier frequencies of interest, the best placement of the probes or human hand, and the optimal grounding connection. Thus, manual calibration and experimentation were required. Generally, instructiondependent leakage occurs on most laptops; key extraction is possible on many laptops, but the requisite signal-to-noise is not always present.

GnuPG. For this case study, we focused on GnuPG version 1.4.15, running on Windows XP and compiled with the MinGW GCC version 4.6.2. This version of GnuPG was the most recent when our research was publicly announced. Following the practice of responsible disclosure, we worked with the authors of GnuPG to suggest several countermeasures and verify their effectiveness against our attacks (including those in [12]; see discussion therein as well as CVE-20134576 [19]). GnuPG 1.4.16, released concurrently with the announcement of our results, contains these countermeasures.

Chosen Ciphertext Injection. Our key extraction attacks require chosen ciphertexts (either adaptive or non-adaptive, depending on the attack). As observed in [12, one way to remotely inject such ciphertexts into GnuPG is to send them as a PGP/MIME-encoded e-mail, to be automatically decrypted by the Enigmail [9] plugin for the Mozilla Thunderbird e-mail client. In the case of the non-adaptive attack, the attacker can provide the chosen ciphertext files to the victim (by any means or guise), and merely needs to conduct the measurement, for a few seconds, when those files are decrypted.

\subsection{Related Work}

Simple and differential power analysis attacks were introduced by Kocher et al. [15, and applied to both symmetric and asymmetric ciphers, implemented on hardware such as smartcards, microcontrollers and FPGAs (see [4, 16, 18, and the references therein).

Clark et al. 6] observed that is it possible to use power analysis to identify, with high probability, the web pages loaded by a web browser on the target 


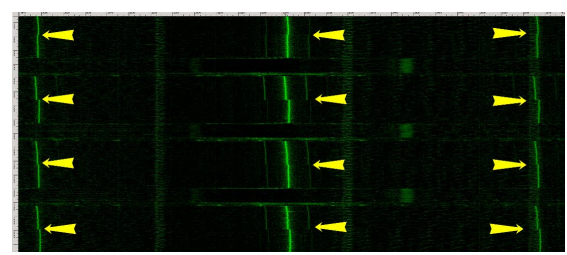

(a) different keys

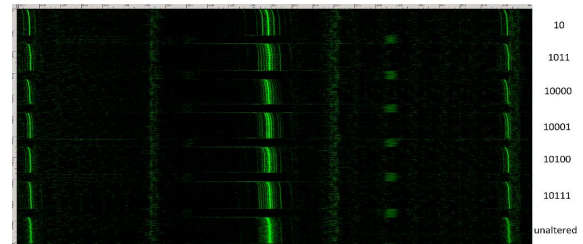

(b) different exponents

Fig. 1. Chassis measurement (1.7 sec, 1.9-2.6 MHz) of a Lenovo 3000 N200. (a) Four GnuPG RSA signatures. The transitions between $p$ and $q$ are marked with yellow arrows. (b) Seven GnuPG RSA decryptions. In the first 6 cases, exponents $\left(d_{p}\right.$ and $d_{q}$ ) are both overridden to be the 2048-bit number obtained by repeating the pattern written to the right. In the last case, the exponent is unaltered. In all cases, the moduli $p$ and $q$ are the same and the ciphertext is set to $n-1$.

machine, by tapping the $\mathrm{AC}$ outlet to which the target is connected. Oren and Shamir [20] observed that the power line voltage on USB ports exhibits a distinct signature when OpenSSL RSA decryption executes, even when the port is disabled; this property is shared by our "far end of cable" channel. Schmidt et al. 22] observed leakage through voltage variations on the I/O pins of embedded devices. Basic multiplication instructions were shown to have operand-specific leakage, in simulation [24] and embedded devices 8] (though this was not demonstrated or exploited on PCs).

The electromagnetic side channel has been studied and exploited for smartcards and FPGA's (e.g., [3, 10, 21]), including for RSA. More recently, Zajic and Prvulovic [27] observed electromagnetic leakage from laptop and desktop computers (but did not show cryptographic applications). Cache attacks were applied to GnuPG's RSA implementation [25].

\section{Computation-Dependent Chassis-Potential Leakage}

The electric potential on a laptop computer's chassis (metal panels, shields and ports) is ideally equal to that of the mains earth ground potential, but in reality it fluctuates greatly. Even when the laptop is grounded (via its power supply or via shielded cables such as Ethernet, USB or VGA), there is non-negligible impedance between the grounding point(s) and other points in the chassis. Voltage, often $10 \mathrm{mV}$ RMS or more 2 develops across this impedance, in part due to currents and electromagnetic fields inside the computer. A natural question is whether information about the ongoing computation can be learned by measuring the chassis potential.

In this section, we show a very simple (yet already troubling) form of leaked information: determining which of several randomly-generated secret keys was

\footnotetext{
${ }^{2}$ After filtering out the strong, but cryptanalytically useless, 50 or $60 \mathrm{~Hz}$ components.
} 
used by the target machine, for a signing or decryption operation. For brevity, in the remainder of this section we discuss chassis potential measurements; similar effects are also present when using the EM side channel.

Figure 1(a) depicts the spectrogram of four GnuPG RSA signing operations, using different 4096-bit random keys (generated beforehand), on a fixed message. Each signing operation is preceded by a short CPU sleep (for visual separation).

The different signing keys can be clearly distinguished by their subtly different spectral signatures (which may itself be of practical interest). Another telling detail is visible: halfway through each signing operation, a transition appears at several frequency bands (marked with yellow arrows). This corresponds to the transition between exponentiation modulo the secret $p$ to exponentiation modulo the secret $q$, in the RSA decryption implementation of GnuPG, which is based on the Chinese Remainder Theorem. We can thus spectrally observe internal, secret-dependent information within the signing operation.

Next, Figure 1(b) demonstrates seven RSA signing operations using different secret exponents which can also be easily distinguished. Finally, similar results are observed for ElGamal decryption, though the exponentiation is shorter and thus its frequency spectrum can be characterized less accurately.

\section{Non-adaptive Attack}

We proceed to describe our cryptanalytic attack techniques (whose applicability will be shown in Section 5). The first technique is a non-adaptive chosen ciphertext attack using very few traces, following the simple power analysis of RSA (see the surveys [4,16,18, and the references therein). We begin by reviewing the high-level modular exponentiation algorithm in GnuPG (Section 3.1), describe our attack (Section 3.2) exploiting this algorithm, and then analyze its success by recalling the inner squaring routines used by GnuPG (Section 3.3) and their behavior under the attack (Section 3.4).

\subsection{GnuPG's Modular Exponentiation Routine}

To perform arithmetic on the large integers occurring in RSA and ElGamal, GnuPG uses an internal mathematical library called MPI (based on GMP [1]). MPI stores large integers as arrays of limbs, i.e., 32-bit words (on x86).

We now review the modular exponentiation routine of GnuPG (as introduced in GnuPG v1.4.14), which is used for both RSA and ElGamal. GnuPG uses a variant of the square-and-multiply modular exponentiation algorithm, processing the bits of the exponent from the most significant bit to the least significant one. To mitigate a cache side-channel attack [25], GnuPG now always performs the multiplication operation in every loop iteration regardless of the exponent's bits (but only uses the result as needed). The pseudocode is given in Algorithm 1 . This top-level exponentiation routine suffices for the high-level description of our attack. For details about GnuPG's underlying squaring routines, necessary for understanding the attack's success, see Sections 3.3 and 3.4 . 


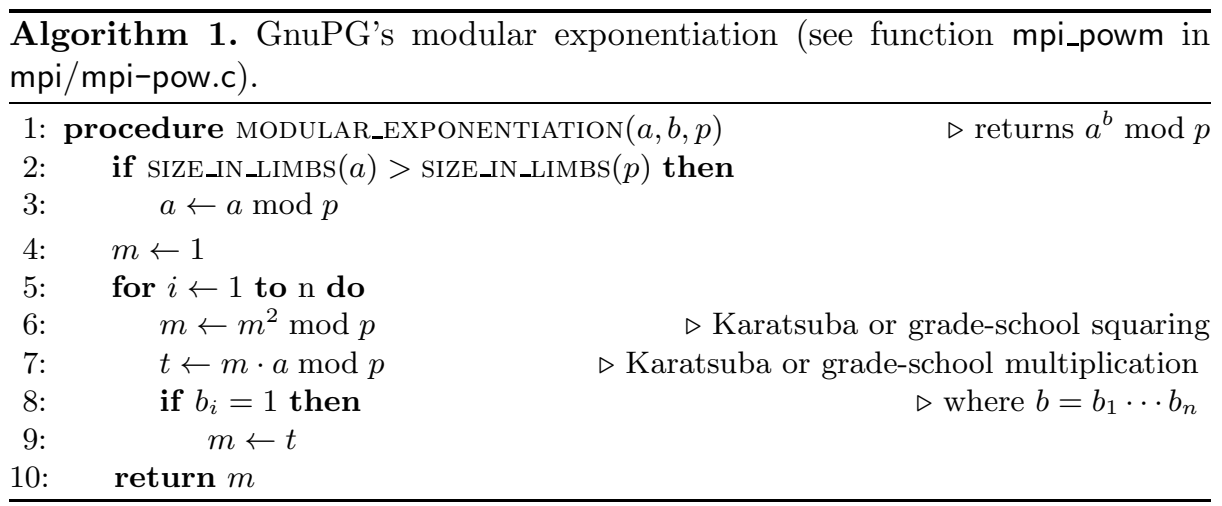

\subsection{The Attack Algorithm}

Since GnuPG 1.4.15 attempts to avoid correlation between the bits of the exponent and high-level operations (such as multiplication), we use a chosen ciphertext attack in order to create correlations between these bits and intermediate values computed inside the low-level operations inside GnuPG's modular exponentiation routine. Moreover, the chosen ciphertext will have an amplification effect, whereby numerous recursive calls will be similarly affected, resulting in a distinct leakage signal over a long time period. This is the key to conducting a $\mathrm{MHz}$-scale attack on a $\mathrm{GHz}$-scale computation.

Following Yen et al. 26] we choose a ciphertext $c$ such that $a \equiv-1(\bmod p)$ during the execution modular_exponentiation. Within GnuPG, this creates a correlation between the bits of the secret exponent $b$ and the number of zero limbs of $m$, as described next. As we will analyze in Sections 3.3 and 3.4 , the number of zero limbs inside $m$ affects the control flow inside the GnuPG basic squaring routine,thus creating discernible differences in the physical leakage.

Note that, for $a \equiv-1(\bmod p)$, the value $m$ during the execution of MODULAR_EXPONENTIATION is always either 1 or -1 modulo $p$. Thus, the value of $m$ in line 7 does not depend on the bits of $b$, and is always 1 modulo $p$ (since $\left.-1^{2} \equiv 1^{2} \equiv 1(\bmod p)\right)$. Consequently, the following correlation holds between the value of $m$ at the start of the $i$-th iteration of the loop in line 5 and $b_{i-1}$.

$-\boldsymbol{b}_{\boldsymbol{i}-\mathbf{1}}=\mathbf{0}$. In this case the branch on line 8 was not taken; thus, the value of $m$ at the start of the $i$-th iteration of the loop in line [5] is also $m=1 \bmod p=1$. Next, since GnuPG's internal representation does not truncate leading zeros, it holds that the value $m$ sent to the squaring routine during the $i$-th iteration contains many zero limbs.

$-\boldsymbol{b}_{\boldsymbol{i}-\mathbf{1}}=\mathbf{1}$. In this case the branch on line 8 was taken, so the value of $m$ at the start of the $i$-th iteration of the loop in line 5 is $m=-1 \bmod p=p-1$. Since $p$ is a random large prime, the value $m$ sent to the squaring routine during the $i$-th iteration contains very few zero limbs. 


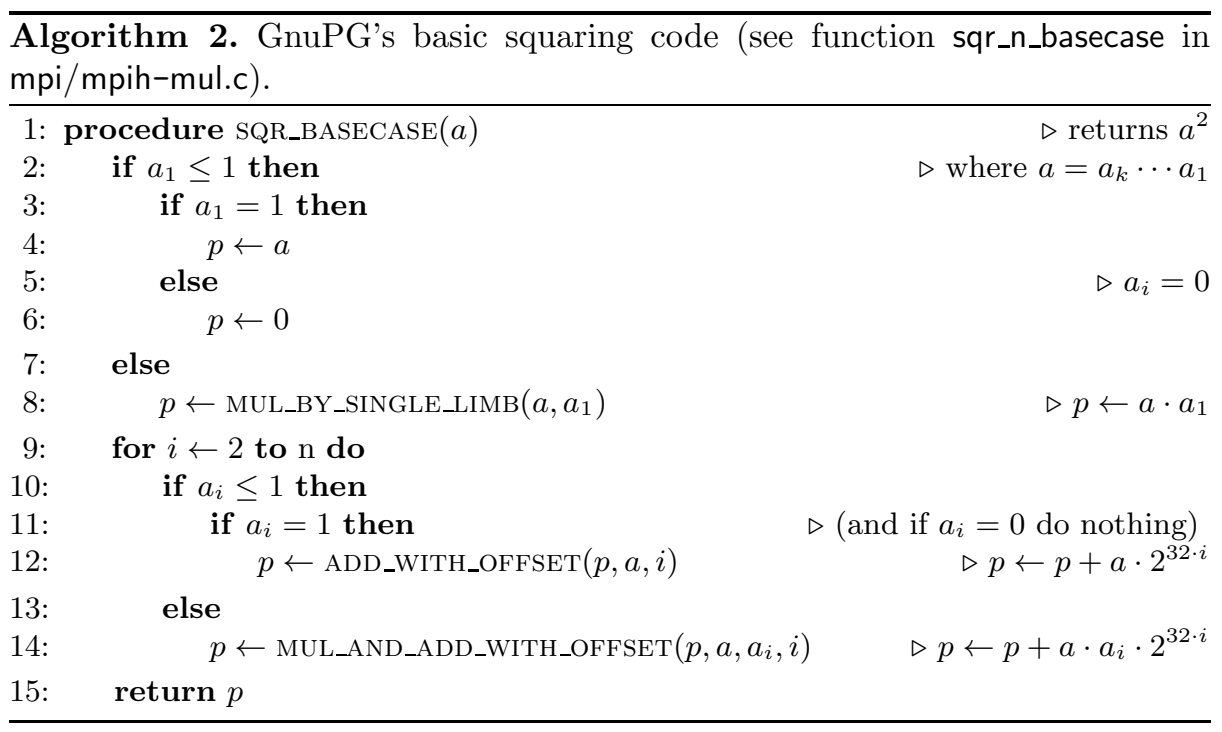

We now proceed to describe the specific ciphertext choices required for our attack for both the RSA and ElGamal case.

Ciphertext Choice for RSA-CRT. Recall that in the case of RSA decryption, GnuPG first computes $c^{d_{p}} \bmod p$ and $c^{d_{q}} \bmod q$, and then combines these via the Chinese Remainder Theorem. By choosing $c=n-1$ where $n=p q$ is the public RSA modulus, the modular reduction in line 3 is always triggered, causing the value of $a$ in line 5 to be $p-1$ (i.e., -1 modulo $p$ as desired).

Ciphertext Choice for ElGamal. For ElGamal encryption, the prime modulus $p$ is part of the public key, so we directly choose the ciphertext to be $p-1$.

\subsection{GnuPG's Squaring Routine}

GnuPG's large-integer squaring routine combines two squaring algorithms: a basic quadratic-complexity squaring routine, and a variant based on a recursive Karatsuba multiplication algorithm [14. The chosen combination of algorithms is based on the size of the operands, measured in whole limbs. We will first discuss the basic squaring algorithm and its key-dependent behavior, and then show how this behavior is preserved by the Karatsuba squaring.

GnuPG's Basic Squaring Routine. The core side-channel weakness we exploit in GnuPG's code lies inside the basic squaring routine. The basic squaring routine used by GnuPG is a quadratic-complexity "grade school" squaring, with optimizations for multiplication by limbs equal to 0 or 1, depicted in Algorithm 2,

Note how SQR_BASECASE handles zero limbs of $a$. When a zero limb of $a$ is encountered, none of the operations MUL_BY_SINGLE_LIMB, ADD_WITH_OFFSET and MUL_AND_ADD_WITH_OFFSET are performed and the loop in line 9 continues 
to the next limb of $a$. Our attack exploits this, by causing the number of such zero limbs to depend on the current bit of the secret exponent, thus affecting the control flow in lines 3 and 11, and thereby the side-channel emanations.

GnuPG's Karatsuba Squaring Routine. The basic squaring routine described above is invoked via two code paths: directly by the modular exponentiation routine (Section 3.1) when the operand is small, and also as the base-case by the Karatsuba squaring routine. The latter is a variant of the Karatsuba multiplication algorithm [14], relying on the following identity:

$$
a^{2}=\left\{\begin{array}{ll}
\left(2^{2 n}+2^{n}\right) a_{\mathrm{H}}^{2}-2^{n}\left(a_{\mathrm{H}}-a_{\mathrm{L}}\right)^{2}+\left(2^{n}+1\right) a_{\mathrm{L}}^{2} & \text { if } a_{\mathrm{H}}>a_{\mathrm{L}} \\
\left(2^{2 n}+2^{n}\right) a_{\mathrm{H}}^{2}-2^{n}\left(a_{\mathrm{L}}-a_{\mathrm{H}}\right)^{2}+\left(2^{n}+1\right) a_{\mathrm{L}}^{2} & \text { otherwise }
\end{array},\right.
$$

where $a_{\mathrm{H}}, a_{\mathrm{L}}$ are the most and least significant halves of $a$, respectively.

\subsection{Attack Analysis}

In this section we analyze the effects of our attack on SQR_BASECASE (Algorithm [2). Recall that in Section 3.2 we created a correlation between the $i$-th bit of the secret exponent $b_{i}$ and the number of zero limbs in the operand $m$ of the squaring routine during iteration $i+1$ of the main loop of the modular exponentiation routine. Concretely, for the case where $b_{i}=1$, we have that $m=-1 \bmod p=p-1$ is a random-looking number containing several thousand bits (2048 bits for the case of RSA and 3072 bits for the case of ElGamal). Conversely, for the case where $b_{i}=0$, we have that $m=1 \bmod p=1$ and, since GnuPG does not truncate leading zeros, the representation of $m$ is a large number (2048 bits for the case of RSA and 3072 bits for ElGamal), all of whose limbs are 0 (except for the least significant).

The code of GnuPG passes $m$ to the Karatsuba squaring routine. For the case where $b_{i}=1$, since $m$ is a random-looking number, this property of $m$ will be preserved in all 3 recursive calls (computing the three squaring operations in Equation (1). Similarly, for $b_{i}=0$, we have that $m=1$, meaning $m_{\mathrm{H}}=0$ and $m_{\mathrm{L}}=1$. Thus, the second case of Equation 1 will always be taken, again preserving the structure of $m$ as having mostly zero limbs, in all 3 recursive calls.

When reaching the recursion's base case, we have the following dependence on $b_{i}$. If $b_{i}=0$, then the values of the operand of SQR_BASECASE during iteration $i+1$ of the main loop of MODULAR_EXPONENTIATION (in all branches of the recursion) will have almost all of their limbs equal to zero. Conversely, if $b_{i}=1$, then the values of the operand of SQR_BASECASE during iteration $i+1$ of the main loop of MODULAR_EXPONENTIATION in all branches of the recursion will be random-looking.

Next, recall the effect of zero limbs in the operand on the code of SQR_BASECASE. Note that the control flow in SQR_BASECASE depends on the number of non-zero limbs in its operand. The drastic change in the number of zero limbs in the operand of SQR_BASECASE is detectable by our side-channel measurements. Thus, we are able to leak the bits of the secret exponent by creating the correlation between 
its bits and the number of zero limbs in the operand of SQR_BASECASE, using our carefully chosen cipher text.

\section{Adaptive Attack}

Our other cryptanalytic technique is an adaptive chosen-ciphertext side-channel attack, which extracts, bit by bit, the prime modulus used during the CRT-based RSA modular exponentiation routine. Our attack on GnuPG was introduced in 12 (following [5]) for the GnuPG acoustic side channel. For self-containment, we give an overview of the attack here. (This attack is not applicable to ElGamal, since the prime modulus $p$ is public.)

The attack recovers the bits of $p=p_{1} \cdots p_{k}$ iteratively, starting with the MSB $p_{1}$. Once we learn all of $p$, we know the factorization of $n$. Moreover, after recovering just the top half of the bits of $p$, it is possible to use Coppersmith's attack [7] to recover the remaining bits.

Ciphertext Choice for RSA. In GnuPG, the MSB is always set, i.e., $p_{k}=1$. Assume that we have already recovered the topmost $i-1$ bits of $p$. To extract the next bit $p_{i}$, we check the two hypotheses about its value, by requesting decryption of an adaptively chosen ciphertext $g^{i, 0}+n$, where $g^{i, 0}=p_{1} \cdots p_{i-1} 10 \cdots 0$ ( $k$ bits in total). Let $n=p q$ be the public RSA modulus. Consider the RSA decryption of $g^{i, 0}+n$. Two cases are possible, depending on $p_{i}$.

$-\boldsymbol{p}_{\boldsymbol{i}}=1$. Then $g^{i, 0}<p$. The ciphertext $g^{i, 0}+n$ is passed as the variable $a$ to Algorithm 1. Since $n=p g$ we have that $g^{i, 0}+n$ has a larger limb count than $p$. This triggers the modular reduction of $a$ in line 3 of Algorithm 1, which returns $g^{i, 0}$, resulting in $a$ being a $k$-bit number having mostly zero limbs. Next, $a$ is passed to the multiplication routine in line 7.

$-\boldsymbol{p}_{\boldsymbol{i}}=\mathbf{0}$. Then $p<g^{i, 0}$ and as in the previous case, $g^{i, 0}+n$ is passed as the variable $a$ to Algorithm 1 triggering the modular reduction of $a$ in line 3 . Since $g^{i, 0}$ and $p$ share the same topmost $i-1$ bits, we have that $g^{i, 0}<2 p$, and the reduction results in $a=g^{i, 0}-q$, which is a $(k-i)$-bit random-looking number. This is then passed to the multiplication routine in line 7

Code Analysis. We now present a high-level analysis of how our bit-by-bit chosen ciphertext attack affects the code of GnuPG. Using the above described ciphertexts, the second operand of the multiplication routine during the entire execution of the main loop of the modular exponentiation routine, will be either full-size and repetitive or shorter and random-looking (depending on $p_{i}$ ).

GnuPG's uses two algorithms for large integer multiplication: the Karatsubabased multiplication algorithm and the grade-school (quadratic time) multiplication algorithm. GnuPG's variant of Karatsuba recursive multiplication relies on the identity $a b=\left(2^{2 n}+2^{n}\right) a_{\mathrm{H}} b_{\mathrm{H}}+2^{n}\left(a_{\mathrm{H}}-a_{\mathrm{L}}\right)\left(b_{\mathrm{L}}-b_{\mathrm{H}}\right)+\left(2^{n}+1\right) a_{\mathrm{L}} b_{\mathrm{L}}$, where $a_{\mathrm{H}}, b_{\mathrm{H}}$ and $a_{\mathrm{L}}, b_{\mathrm{L}}$ are the most and least significant halves of $a$ and $b$, respectively. We thus see that GnuPG's Karatsuba multiplication preserves the structure of $a$ as being either random-looking or containing many zero limbs. That is, if $a$ is random-looking, then $a_{\mathrm{H}} b_{\mathrm{H}},\left(a_{\mathrm{H}}-a_{\mathrm{L}}\right)\left(b_{\mathrm{L}}-b_{\mathrm{H}}\right)$ and $a_{\mathrm{L}} b_{\mathrm{L}}$ are 
random-looking as well. Conversely, if a contains mostly zero limbs, the values of $a_{\mathrm{H}} b_{\mathrm{H}},\left(a_{\mathrm{H}}-a_{\mathrm{L}}\right)\left(b_{\mathrm{L}}-b_{\mathrm{H}}\right)$ and $a_{\mathrm{L}} b_{\mathrm{L}}$ also contain mostly zero limbs.

Next, when the recursion reaches its base case, GnuPG passes $a$ to the basic multiplication routine which is implemented similarly to Algorithm 2, In particular, it includes optimizations for zero limbs similar to lines 3 and 11. Thus, we are able to leak the bits of $p$, one bit at a time, by creating a correlation between the current bit of $p$ and the number of zero limbs in the second operand of GnuPG's basic multiplication routine using our chosen ciphertexts.

The above is a high-level description of the adaptive attack. In order to achieve full RSA key extraction, improvements are required to the basic attack algorithm. See 12 for details.

\section{Empirical Key-Extraction Results}

\subsection{Chassis-Potential Attack}

\subsubsection{Setup}

As discussed in Section 2 there are computation-dependent fluctuations of the electric potential on the chassis of laptop computers. We measured the electric potential of the laptop's chassis by touching it with the simplest possible probe: a plain wire, $80 \mathrm{~cm}$ long. The wire is pressed against the chassis by hand, or (for longer attacks) using an alligator clip. The wire's potential is then measured through an amplifier, filters, and a digitizer.

Grounding. The attack measures the voltage between the room's mains earth ground potential and the target computer's chassis potential. Put otherwise, we create a ground loop which includes the laptop chassis and the amplifier, and then measure the voltage across the amplifier's input impedance (and thus its complement: the voltage across the laptop chassis). Thus, correct grounding is essential to maximizing the signal-to-noise ratio. The measurement is done in single-ended mode, in reference to the mains earth ground potential, using lowimpedance ground connection to the amplifier and digitizer. The target laptop is grounded through one of its shielded I/O ports (we used a VGA cable to a grounded screen, or a USB cable to a grounded printer). If the target laptop's grounding is removed, but the laptop is still connected to 3-prong (grounded) AC-DC power supply, the signal-to-noise ratio typically degrades. With a 2prong (ungrounded) power supply and no other ground connections, the signalto-noise ratio is very low and our attacks do not work.

Chassis Probe Placement. The chassis of modern laptops, while made mostly of metal (for EMI shielding), is typically covered with non-conductive plastic. However, many IO ports, such as USB, Ethernet, VGA and HDMI, typically have metal shielding which is connected to the chassis or PCB ground, and thus can be probed by the attacker. Also, metal heatsink fins are often easily reachable through the exhaust fan grill. Heuristically, the best results are achieved when the chassis is probed close to the CPU and its associated voltage 
regulator circuitry, and if the laptop's ground connection is distant from the probing point.

Chassis Potential or EM? To ascertain that we were indeed measuring chassis potential rather than stray electromagnetic fields, we broke the direct galvanic connection between the probe wire and the laptop's chassis, by inserting a sheet of paper in-between. This always resulted in severe signal attenuation.

\subsubsection{Non-adaptive Chassis-Potential Attack}

MF Measurement Equipment. The non-adaptive attack exploits signals in the Medium Frequency (MF) frequency band, on the order of $2 \mathrm{MHz}$. To measure these signals, we connected the probe wire to a $16 \mathrm{kHz}$ high-pass filter, followed by a high-input-impedance low-noise amplifier (Femto HVA-200M-40-F, $40 \mathrm{~dB}$ gain). The amplified signal was then low-pass filtered at $5 \mathrm{MHz}$ and digitized at 12 bits and $10 \mathrm{Msample/sec} \mathrm{(National} \mathrm{Instruments} \mathrm{PCI} \mathrm{6115).}$

Analyzing the Signal. The signals presented in Figure 1(b) provide the first indication as to how the different exponents might be distinguished. For periodic exponents, the leakage signal spectrum takes the form of small, distinct side lobes centered around a dominant frequency peak. This indicates that the bits of the exponents manifest themselves as modulations on a central sinusoidal carrier wave. Further analysis reveals that the carrier is frequency-modulated, meaning that its instantaneous frequency changes slightly in accordance with the current bit of the exponent. Thus, in order to recover these bits, we obtain the dominant instantaneous frequency as a function of time, by applying FM demodulation digitally. The signal is first filtered using a $30 \mathrm{kHz}$ band-pass filter centered at the carrier frequency. Next, the signal is demodulated using a Discrete Hilbert Transform. Additional filtering is then performed on the demodulated signal, to suppress high frequency noise and to compensate for a slow frequency drift of the carrier wave. Figure 2(a) shows an example of a fully demodulated leakage signal; the correlation with the secret key bits can clearly be seen.

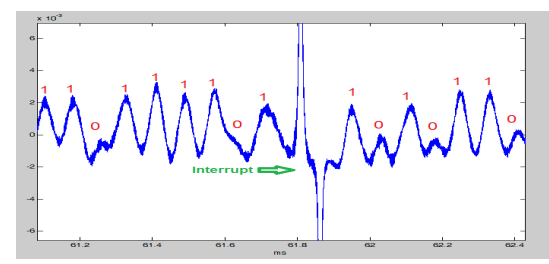

(a) A segment of the demodulated signal. Note the correlation between the signal and the secret key bits.

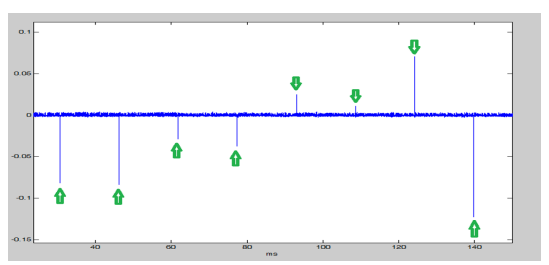

(b) Demodulation of the entire signal. The interrupts, occurring every $15 \mathrm{~ms}$, are marked by green arrows.

Fig. 2. Frequency demodulation of the leakage signal during a decryption operation using a randomly generated 4096-bit RSA key 
Key Extraction. Theoretically, it should be possible to extract the entire secret key from a single demodulated trace, provided the measurement is robust and has a high signal-to-noise ratio. However, we observed a periodic interrupt (marked in Figure 2(a)), which disrupted the key extraction. The interrupt manifests as a large frequency fluctuation in the carrier every 15 milliseconds (Figure 2(b)], corresponding to the $64 \mathrm{~Hz}$ timer interrupt frequency on the target laptop. These interrupts systematically occur at the same time during the decryption process, and thus disrupt similar bit offsets in repeated decryptions. Fortunately, the inherent jitter is sufficient so that, over a few measurements, every bit is (with high probability) undisturbed in some sample. Conversely, jitter creates a difficulty in aligning the multiple traces. We thus break each trace, post-modulation, into multiple time segments. The segments are then aligned via correlation, and averaged. This results in an interrupt-free aggregate trace, with very high SNR. The bits are then extracted using a peak detection algorithm.

Non-adaptive RSA Key Extraction. Applying our non-adaptive attack on a randomly generated 4096-bit RSA key while measuring the chassis potential of a Lenovo 3000 N200 laptop during 6 decryption operations, each lasting 0.35 sec, we have directly extracted 2044 out of 2048 bits of $d_{p}$ thereby extracting the key. The laptop was powered by a 3-prong AC-DC power supply (Lenovo, 90W, model 42T4428), without additional connections.

Non-adaptive ElGamal Key Extraction. Attacking the exponentiation in ElGamal decryption, and applying similar cryptanalytic and signal analysis, we extracted all but 2 of the bits of the secret exponent from a randomly generated 3072-bit ElGamal key by measuring the chassis potential of a Lenovo 3000 N200 laptop during 9 decryption operations, each lasting $0.1 \mathrm{sec}$

\subsubsection{Adaptive Chassis-Potential Attack}

We now discuss results obtained using the adaptive attack described in Section 4. While this attack requires more decryption operations in order to recover the key, it utilizes lower frequency signals and requires much lower signal-to-noise ratio than the non-adaptive attack.

VLF/LF Measurement Equipment. The adaptive attack can exploit very low bandwidth signals, in the VLF and LF frequency bands: in the order of $15-40 \mathrm{kHz}$ (depending on the laptop model). To measure these signals, we used a more compact, higher dynamic range, measurement chain. The probe was directly connected to a high-input-impedance low-noise amplifier (customized Brüel\&Kjær 5935, usually set to $40 \mathrm{~dB}$ gain). The amplified signal was highpass filtered at $10 \mathrm{kHz}$, and digitized at 16 bits and $200 \mathrm{Ksample} / \mathrm{sec}$ (National Instruments MyDAQ).

Analyzing the Leakage Signal. Recall that GnuPG's RSA code performs modular exponentiation modulo $p$ followed by a modular exponentiation modulo $q$. Figure 3(a) shows a typical recording of RSA decryption when the value of the attacked bit of $q$ is 0 , and Figure $3(\mathrm{~b})$ shows a recording of RSA decryption 


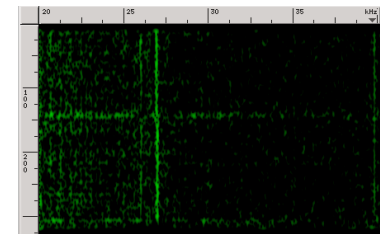

(a) attacked bit is $q_{i}=0$

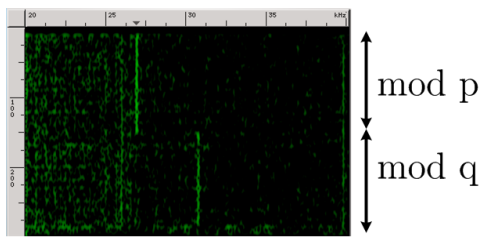

(b) attacked bit is $q_{i}=1$

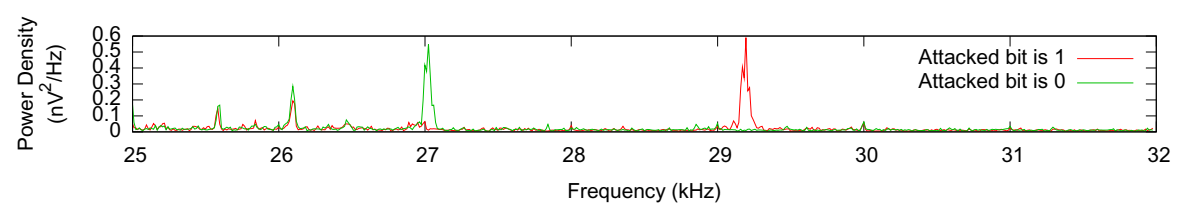

(c) Frequency spectra of the second modular exponentiation

Fig. 3. Chassis measurement of RSA decryption for various values of the attacked bit executed on a Lenovo ThinkPad T60

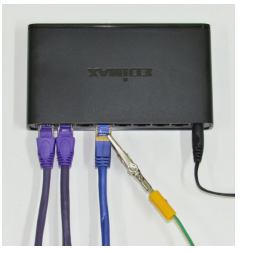
(a) Measuring shield potential at the far side of an Ethernet cable (probed at the Ethernet switch).

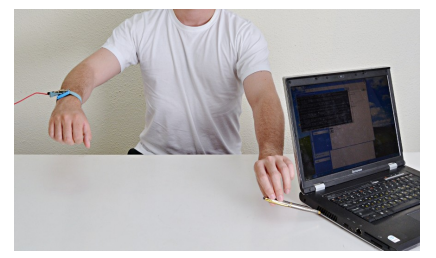

(b) Non-adaptive "human touch" attack through a metal pen touching the heatsink fins. The wristband is connected to the probe wire.

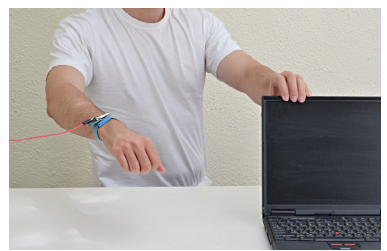

(c) Adaptive "human touch" attack by bare hand. The wristband is connected to the probe wire.

Fig. 4. "Far end of cable" and "human touch" attacks

when the value of the attacked bit of $q$ is 1 . Several effects are shown in the figures. As in figure 1(a) the transition between $p$ and $q$ is clearly visible. Note, then, that the signatures of the modular exponentiation using the prime $q$ (the second exponentiation) are quite different in Figures $3(\mathrm{a})$ vs. $3(\mathrm{~b})$. This can be seen more clearly in Figure 3(c), which summarizes the aforementioned spectral signatures by taking the median, over time, for each. This clear difference is used to extract the bits of $q$, as explained in Section 4 .

Adaptive RSA Key Extraction. By connecting the VLF/LF measurement setup to a Lenovo ThinkPad T60, powered by the 3-prong AC-DC power supply without additional connections 3 we directly extracted the 1024 most significant

\footnotetext{
${ }^{3}$ Grounding the laptop to mains earth, via some port, would improve the signal quality
} (see Section 5.1.1); but the adaptive attack is sufficiently robust to not require this. 
bits of the secret prime $q$ of a randomly-generated 4096-bit RSA key in approximately 1 hour. By Coppersmith's technique, this results in full key extraction. (Alternately, it is possible to continue the attack and recover remaining bits.)

\section{2 "Far End of Cable" Attack}

The electric potential on a laptop's chassis and external ports can measured from far away. When a cable is plugged into one of the laptop's IO ports (such as USB, Ethernet and VGA), the port's shield typically contacts a plug shield, which in turn is connected to a conductive cable shield running the length of the cable. Thus, one can measure the chassis potential from the far side of cables connected to the aforementioned ports. Ethernet cables, for examples, often span long distances, across and between building floors. An attacker who gains access to the far side of the cable (see Figure 4(a), or taps the shield along the way, can measure the approximate chassis potential.

The attack does not utilize data transmitted over the cable, and is oblivious to whether the port is even enabled.

We conducted the attacks by connecting the target laptop, through a 10meter long shielded CAT5e Ethernet cable, to an Ethernet switch (EDIMAX ES-3308P). We attached a plain wire to the cable shield via a clip, on the switch side (see Figure 4(a) and measured its potential.

Non-adaptive RSA Key Extraction. Using the MF measurement setup (see Section 5.1.2), we measured the chassis potential of a Lenovo 3000 N200 laptop through the shield of the Ethernet cable. The laptop was powered by the 3 -prong AC-DC power supply, and was also connected via a shielded VGA cable to a grounded monitor (Dell 2412M). We directly extracted 2042 out of 2048 bits of $d_{p}$, by observing the shield's potential during 5 decryption operations (each lasting $0.35 \mathrm{sec}$ ). Similar results were obtained at the far side of a USB cable.

Non-adaptive ElGamal Key Extraction. Applying a similar attack to ElGamal decryption, we extracted all but 3 of the bits of the secret exponent, from a randomly-generated 3072-bit ElGamal key, by observing the shield's potential during 4 decryption operations (each lasting $0.1 \mathrm{sec}$ ).

Adaptive RSA Key Extraction. We attacked a Lenovo ThinkPad T60 laptop, through an Ethernetor USB cable, whose shield potential was measured using the VLF/LF measurement equipment (see Section 5.1.3). The laptop was powered by the 3-prong AC-DC power supply, without additional connections. In this setting, we extracted the 1024 most significant bits of the secret prime $q$ (and thus, by Coppersmith's technique, full key extraction) in 1 hour.

\section{3 "Human Touch" Attack}

On many laptops, the chassis potential can be sensed indirectly through a human body. An attacker can sense the chassis potential by merely touching a conductive part of the laptop chassis with his hand. This affects the electric potential 
of the attacker's body (assuming suitable insulation, e.g., nonconductive floor or shoes). Surreptitiously, the attacker can measure the electric potential induced on his own body, using a concealed probe, in reference to some nearby conductive grounded surface in the room. Even this circuitous measurement, through an ill-characterized, high-impedance path, can suffice for key extraction.

Non-adaptive RSA Key Extraction. Using the MF measurement setup (see Section 5.1.2), we measured the chassis potential of a Lenovo 3000 N200 laptop indirectly, though the body of a volunteer (one of the authors). The volunteer held a paperclip or a metal pen in his hand, and briefly touched it to the laptop's heatsink fins, which are easily reachable through the exhaust vent. Concurrently, the volunteer's body potential was measured using the MF measurement equipment (see Section 5.1.2), via a probe wire attached to a conductive wristband on his other wrist (see Figure 4(b)).

The laptop was powered by the 3-prong AC-DC power supply, with no further connection. Applying our non-adaptive attack and the signal analysis techniques from Section 5.1 .2 on a randomly generated 4096-bit RSA key, we directly extracted 2042 out of 2048 bits of $d_{p}$ by observing the volunteer's body potential (while holding the paperclip against the laptop's heatsink) during 6 decryption operations, each lasting $0.35 \mathrm{sec}$.

Non-adaptive ElGamal Key Extraction. Applying our non-adaptive attack and the signal analysis techniques from Section 5.1.2 on a randomly generated 3072-bit ElGamal key, we were able to extract all but 3 of the bits of the secret exponent by observing the volunteer's body potential (while holding the paperclip against the laptop's heatsink) during 16 decryptions, each lasting $0.1 \mathrm{sec}$.

Adaptive RSA Key Extraction. The adaptive attack, being more robust (due to relying on lower-frequency, longer-duration signals), succeeded with unaided finger touch. A patient volunteer touched the chassis (specifically, VGA connector shield) of a Lenovo ThinkPad T61 with his fingers. The volunteer's body potential was measured using the VLF/LF measurement equipment (see Section 5.1.3), through the aforementioned wristband, and the laptop was connected as above. On some ThinkPad models, this attack can even be mounted by simply touching the rubber-coated LCD cover (see Figure 4(c)).

In this setting, we directly extracted the topmost 1024 bits of the prime $q$ of a randomly generated 4096-bit RSA key from in 1 hour, thereby (via Coppersmith's technique) completely extracting the key.

\subsection{Electromagnetic (EM) Attack}

Next, we studied EM emanations from laptop computers, in the MF band (approximately $2 \mathrm{MHz}$ ). We used a near-field magnetic probe (Rohde\&Schwarz

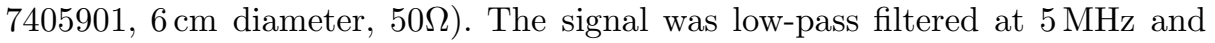
amplified using a low-noise amplifier (a customized Mini-Circuits ZPUL-30P). This is digitized at 12 bits and $10 \mathrm{Msample/sec} \mathrm{(NI} \mathrm{PCI} \mathrm{6115).}$ 
EM Probe Placement. The placement of the EM probe greatly influences the measured signal and noise. We wish to measure EM emanations close to the CPU's voltage regular, located on the laptop's motherboard, yet without mechanical intrusion. Concretely, the rear-left corner often yields the best signal.

Non-adaptive RSA Key Extraction. Applying our non-adaptive attack to a 4096-bit RSA key, we directly extracted 2046 out of 2048 bits of $d_{p}$, by measuring the EM emanations from a Lenovo 3000 N200 laptop during 5 decryption operations, each lasting about $0.35 \mathrm{sec}$.

Non-adaptive ElGamal Key Extraction. Using the same experimental setup and applying our non-adaptive attack to a randomly generated 3072-bit ElGamal key, we were able to extract all but 3 of the bits of the secret exponent by measuring the EM emanations from a Lenovo 3000 N200 laptop during 16 decryption operations, each lasting about $0.1 \mathrm{sec}$.

\subsection{Power Analysis Attack}

Finally, we revisited the classic power analysis channel, and analyzed the current draw fluctuations on the power supply of the target laptop in the VLF/LF frequency bands. Specifically, we placed a $0.5 \Omega$ resistor in series with the laptop's power supply, on the low ("ground") supply rail.

Adaptive RSA Key Extraction. We measured the voltage on the resistor using a National Instruments MyDAQ device though a $150 \mathrm{kHz}$ low-pass filter. We directly extracted the topmost 1024 bits of the prime $q$ of a randomly generated 4096-bit RSA key, from a Lenovo ThinkPad T61, in one hour.

\section{Conclusion}

While physical side-channel attacks have proven very effective at breaking cryptosystems, most research attention has focused on small and relatively simple devices. This paper demonstrated that PC systems too are vulnerable, despite their complexity, noise, and challenging electrical characteristics. Moreover, PCs can be attacked by mere touch or from afar by almost any wired connection.

Following our observations, several software countermeasures were proposed and incorporated into GnuPG 1.4.16 and libgcrypt 1.6. While these countermeasures indeed foil the attacks presented in this paper, the key distinguishing attack is unaffected and still present in the latest versions of GnuPG; mitigating it in software, without a large overhead, remains an open problem.

Physical mitigation techniques include Faraday cages (against EM attacks), insulating enclosures (against chassis and touch attacks), and photoelectric decoupling or fiberoptic connections (against "far end of cable" attacks). However, inexpensive protection of PCs appears difficult, especially for the chassis channel. The common method for filtering conducted emanations on power supply lines is to use bypass capacitors to shunt stray AC currents into the ground, but 
this obviously does not apply to the ground line itself. Robust low-impedance grounding and shielding, with careful attention to current paths, should reduce voltages across the ground and chassis (at costs in engineering effort and portability). We conjecture that prudent design of switching power supplies can reduce computation-dependent leakage without significantly hampering efficiency.

Acknowledgments. We are indebted to Adi Shamir for insightful discussions and suggestions, and to Lev Pachmanov for writing much of the software setup used in our experiments. Ezra Shaked assisted in constructing and configuring the experimental setup. Assa Naveh assisted in various experiments, and offered valuable suggestions. Sharon Kessler provided copious editorial advice.

This work was sponsored by the Check Point Institute for Information Security; by European Union's Tenth Framework Programme (FP10/2010-2016) under grant agreement 259426 ERC-CaC, by the the Leona M. \& Harry B. Helmsley Charitable Trust; by the Israeli Ministry of Science and Technology; by the Israeli Centers of Research Excellence I-CORE program (center 4/11); and by NATO's Public Diplomacy Division in the Framework of "Science for Peace".

\section{References}

1. GNU multiple precision arithmetic library, http://gmplib.org/

2. The GNU Privacy Guard, http://www.gnupg.org

3. Agrawal, D., Archambeault, B., Rao, J.R., Rohatgi, P.: The EM side-channel(s). In: Kaliski Jr., B.S., Koç, Ç.K., Paar, C. (eds.) CHES 2002. LNCS, vol. 2523, pp. 29-45. Springer, Heidelberg (2003)

4. Anderson, R.J.: Security engineering - a guide to building dependable distributed systems, 2nd edn. Wiley (2008)

5. Brumley, D., Boneh, D.: Remote timing attacks are practical. Computer Networks 48(5), 701-716 (2005)

6. Clark, S.S., Mustafa, H., Ransford, B., Sorber, J., Fu, K., Xu, W.: Current events: Identifying webpages by tapping the electrical outlet. In: Crampton, J., Jajodia, S., Mayes, K. (eds.) ESORICS 2013. LNCS, vol. 8134, pp. 700-717. Springer, Heidelberg (2013)

7. Coppersmith, D.: Small solutions to polynomial equations, and low exponent RSA vulnerabilities. J. Cryptology 10(4), 233-260 (1997)

8. Courrège, J.-C., Feix, B., Roussellet, M.: Simple power analysis on exponentiation revisited. In: Gollmann, D., Lanet, J.-L., Iguchi-Cartigny, J. (eds.) CARDIS 2010. LNCS, vol. 6035, pp. 65-79. Springer, Heidelberg (2010)

9. Enigmail Project, T.: Enigmail: A simple interface for OpenPGP email security, https://www.enigmail.net

10. Gandolfi, K., Mourtel, C., Olivier, F.: Electromagnetic analysis: Concrete results. In: Koç, Ç.K., Naccache, D., Paar, C. (eds.) CHES 2001. LNCS, vol. 2162, pp. 251-261. Springer, Heidelberg (2001)

11. Genkin, D., Shamir, A., Tromer, E.: RSA key extraction via low-bandwidth acoustic cryptanalysis (extended version). IACR Cryptology ePrint Archive 2013, 857 (2013), extended version of [12]

12. Genkin, D., Shamir, A., Tromer, E.: RSA key extraction via low-bandwidth acoustic cryptanalysis. In: Garay, J.A., Gennaro, R. (eds.) CRYPTO 2014, Part I. LNCS, vol. 8616, pp. 444-461. Springer, Heidelberg (2014), See [11] for extended version 
13. Hu, W.M.: Lattice scheduling and covert channels. In: Proceedings of the IEEE Symposium on Security and Privacy, pp. 52-61 (1992)

14. Karatsuba, A., Ofman, Y.: Multiplication of Many-Digital Numbers by Automatic Computers. Proceedings of the USSR Academy of Sciences 145, 293-294 (1962)

15. Kocher, P., Jaffe, J., Jun, B.: Differential power analysis. In: Wiener, M. (ed.) CRYPTO 1999. LNCS, vol. 1666, pp. 388-397. Springer, Heidelberg (1999)

16. Kocher, P., Jaffe, J., Jun, B., Rohatgi, P.: Introduction to differential power analysis. Journal of Cryptographic Engineering 1(1), 5-27 (2011)

17. Kuhn, M.G.: Compromising emanations: Eavesdropping risks of computer displays. $\mathrm{PhD}$ dissertation (2003)

18. Mangard, S., Oswald, E., Popp, T.: Power analysis attacks — revealing the secrets of smart cards. Springer (2007)

19. MITRE: Common vulnerabilities and exposures list, entry CVE-2013-4576 (2013), http://cve.mitre.org/cgi-bin/cvename.cgi?name=CVE-2013-4576

20. Oren, Y., Shamir, A.: How not to protect PCs from power analysis (2006), http://iss.oy.ne.ro/HowNotToProtectPCsFromPowerAnalysis, CRYPTO rump session

21. Quisquater, J.J., Samyde, D.: Electromagnetic analysis (EMA): Measures and counter-measures for smart cards. In: E-smart 2001, pp. 200-210 (2001)

22. Schmidt, J.-M., Plos, T., Kirschbaum, M., Hutter, M., Medwed, M., Herbst, C.: Side-channel leakage across borders. In: Gollmann, D., Lanet, J.-L., IguchiCartigny, J. (eds.) CARDIS 2010. LNCS, vol. 6035, pp. 36-48. Springer, Heidelberg (2010)

23. Tokunaga, C., Blaauw, D.: Securing encryption systems with a switched capacitor current equalizer. IEEE Journal of Solid-State Circuits 45(1), 23-31 (2010)

24. Walter, C.D., Samyde, D.: Data dependent power use in multipliers. In: IEEE Symposium on Computer Arithmetic, pp. 4-12 (2005)

25. Yarom, Y., Falkner, K.E.: Flush+reload: a high resolution, low noise, L3 cache side-channel attack. IACR Cryptology ePrint Archive 2013, 448 (2013)

26. Yen, S.-M., Lien, W.-C., Moon, S.-J., Ha, J.: Power analysis by exploiting chosen message and internal collisions - vulnerability of checking mechanism for RSAdecryption. In: Dawson, E., Vaudenay, S. (eds.) Mycrypt 2005. LNCS, vol. 3715, pp. 183-195. Springer, Heidelberg (2005)

27. Zajic, A., Prvulovic, M.: Experimental demonstration of electromagnetic information leakage from modern processor-memory systems. IEEE Transactions on Electromagnetic Compatibility (to appear) 